\title{
Speckle-pattern visibility of light transmitted through a multimode optical fiber
}

\author{
B. Crosignani, B. Daino, and P. Di Porto \\ Fondazione Ugo Bordoni, Istituto Superiore P.T., Rome, Italy \\ (Received 7 June 1976)
}

\begin{abstract}
We show that the reduction in visibility of the speckle pattern obtained from laser light that has been passed through a step-index light guide can be found from a knowledge of the model dispersion of the guide, and the bandwidth of the laser beam.
\end{abstract}

In a recent letter, ${ }^{1}$ some interesting results have been published on the visibility of the speckle pattern produced by coherent light after propagation in a light guide. The author investigates the dependence of the speckle visibility on the guide length for a step-index and a SELFOC fiber, using a He-Ne laser as an exciting source. The most significant result is the fast decay of the visibility after few centimeters for the stepindex fiber, while such a decay is not observed over the same length of SELFOC fiber. We wish to present here a simple interpretation of this effect in terms of the coherence time $t_{c}$ (or of the spectral bandwidth $\delta f \simeq 1 / t_{c}$ ) of the source and of the modal dispersion of the guide. In previous works, ${ }^{2,3}$ it has been shown that the smearing out of the speckle pattern on the exit face of the fiber is related to the spectral bandwidth of the source and to the propagation length $L$. More precisely, two modes do not interfere at the exit face of the fiber when the traveled distance is such that their relative delay exceeds the coherence time of the source. After this distance, the total pattern is given by the sum of the intensities of the different modes, which gives rise to the smoothing of the speckle pattern.

In a step-index guide, the time delay $\tau$ between the straight ray and the one propagating at the critical angle is given by

$$
\tau=L n_{1}\left(n_{1}-n_{2}\right) /\left(c n_{2}\right),
$$

$n_{1}$ and $n_{2}$ being, respectively, the refractive index of the core and of the surrounding medium. Thus, the

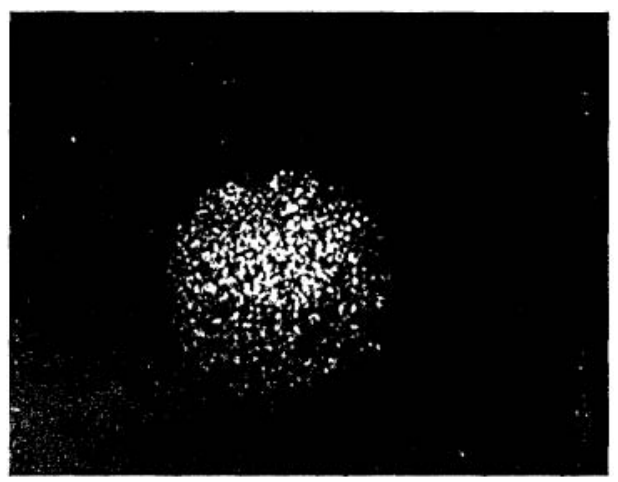

FIG ${ }^{\circ}$. Speckle pattern on the exit face of a fiber $30 \mathrm{~m}$ long, $\dot{4} 5 \mu \mathrm{m}$ in diameter, and with $n_{1} \simeq 1.54, n_{2} \simeq 1.51$, for a bandwidth $\delta f=0.2 \mathrm{GHz}$.

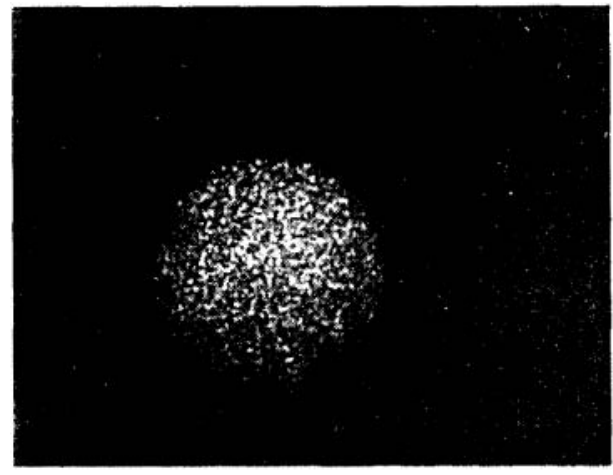

FIG. 2. Speckle pattern for $\delta f=0.5 \mathrm{GHz}$.

length $L_{c}$ at which the two rays do not interfere anymore is

$$
L_{c}=\frac{c n_{2} t_{c}}{n_{1}\left(n_{1}-n_{2}\right)} \simeq \frac{c n_{2}}{n_{1}\left(n_{1}-n_{2}\right) \delta f} .
$$

Assuming, for the experiment reported in Ref. 1, $n_{1} \simeq 1.5, n_{2} \simeq 1, \delta f \simeq 1.5 \mathrm{GHz}$, we obtain $L_{c} \simeq 27 \mathrm{~cm}$, which is of the same order of magnitude of the observed decay length. Of course, the same argument assures that this effect is not observable on the lengths of SELFOC fiber employed in the experiment. In fact, due to the small modal dispersion of this kind of fiber, $L_{c}$ fairly exceeds $50 \mathrm{~cm}$.

We wish finally to observe that the effect of progressive disappearance of the speckle pattern in a fiber of



FIG. 3. Speckle pattern for $\delta f=2 \mathrm{GHz}$. 


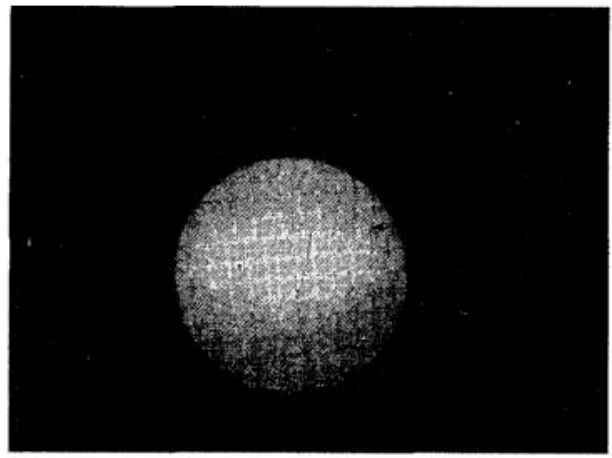

FIG. 4. Speckle pattern for $\delta f=10 \mathrm{GHz}$. fixed length for increasing source bandwidth has been used for measuring modal dispersion in a step-index fiber (see Figs. 1-4). ${ }^{4}$

${ }^{1}$ H. Takahara, Appl. Opt. 15, 609 (1976).

${ }^{2}$ B. Crosignani and P. Di Porto, J. Appl. Phys. 44, 4616 (1973).

${ }^{3}$ B. Crosignani, B. Daino, and P. Di Porto, Opt. Commun. 11, 178 (1974).

${ }^{4} \mathrm{~B}$. Crosignani and P. Di Porto, in Proceedings of the SPIE/ SPSE Technical Symposium East, Vol. 77 (Reston, Virginia, 1976). 